\title{
The anti-lung cancer effect of ethyl acetate extract from Wenxia Formula in vivo via the Hedgehog-Gli1 signaling pathway mediated by cancer associated fibroblasts
}

\author{
Meng Wang ${ }^{1,2}$, Qian-Yu Bi ${ }^{1}$, Ya-Nan Zhang ${ }^{1}$, Xu-Ming ji ${ }^{3}$
}

\author{
${ }^{1}$ College of Chinese Traditional Medicine, Shandong University of Traditional Chinese \\ Medicine, Jinan, Shandong, China \\ ${ }^{2}$ Department of Medicine, Jining No. 1 People's Hospital, Jining, Shandong, China \\ ${ }^{3}$ School of Basic Medical Sciences, Zhejiang Chinese Medical University, Hangzhou, \\ Zhejiang, China
}

Submitted: 3 March 2020

Accepted: 24 May 2020

Arch Med Sci

DOI: https://doi.org/10.5114/aoms.2020.100106

Copyright @ 2020 Termedia \& Banach

\section{Abstract}

Introduction: Previous research had shown that the ethyl acetate extract from Wenxia Formula (WFEA extract) had significant effects in inhibiting tumor and cancer associated fibroblasts (CAFs). However, the anti-cancer mechanism of the WFEA extract was still unclear. In this study, we aimed to illuminate the possible anti-cancer mechanisms of the WFEA extract.

Material and methods: Ultra-performance liquid chromatography quadrupole time-of-flight mass spectrometry (UPLC/Q-TOF-MS) was applied to analyze and identify the constituents of WFEA extract. The lung cancer xenograft model was conducted to explore the antitumor activity of WFEA extract by measuring tumor volume and weight, HE staining, immunohistochemistry, and immunofluorescence assay. Western blot and qRT-PCR were used to measure the expressions of related proteins and genes in the HedgehogGli1 (Hh-Gli1) signaling pathway.

Results: A total of 15 chemical constituents were identified in the WFEA extract. The xenograft model showed that WFEA extract could inhibit tumor growth and had no significant effect on body weight of mice. In addition, WFEA extract reduced the expression of $\alpha$-SMA, FAP and $N$-cadherin in the CAF mediated lung cancer xenograft model and increased the expression of E-cadherin. Western blot and qRT-PCR showed that WFEA extract reduced the expression of related proteins and genes (Smo, Gli1 and Ptch1) in the Hh-Gli1 signaling pathway.

Conclusions: WFEA extract had a certain inhibitory effect on lung cancer, and its mechanism may be related to the CAF mediated Hh-Gli1 signaling pathway.

Key words: Wenxia Formula, WFEA extract, cancer associated fibroblasts, ultra-performance liquid chromatography quadrupole time-of-flight mass spectrometry, Hh-Gli1.

\section{Introduction}

Lung cancer is one of the most common malignancies in the world. In 2012, a total of 1.8 million new cancer cases and 1.6 million related deaths were reported worldwide [1]. Approximately $10-15 \%$ of lung can-

\author{
Corresponding author: \\ Prof. Xu-Ming Ji \\ School of Basic Medical \\ Sciences \\ Zhejiang Chinese Medical \\ University \\ No. 548, Binwen Road \\ Hangzhou, Zhejiang \\ 310053, China \\ Tel: +86-18615179139 \\ E-mail: jixuming724@163. \\ com
}


cers are small cell lung cancers, and about $85 \%$ are non-small cell lung cancers, including adenocarcinoma, large cell carcinoma, squamous cell carcinoma, etc. [2]. The 5-year survival rate of lung cancer is currently only $16 \%$, and more than $70 \%$ of patients are diagnosed with an advanced stage due to the lack of early clinical symptoms [3].

In traditional Chinese medicine (TCM), the formation of cancer is considered to be caused by the infiltration of a pathogen based on a deficiency of vital-qi $[4,5]$. TCM can inhibit tumor growth and metastasis through overall regulation of the tumor microenvironment, thereby regulating and improving the clinical symptoms of patients, improving patients' quality of life and extending their survival [6]. Therefore, the regulatory effect of TCM on the tumor microenvironment has become one of the main research directions of TCM against tumors. The tumor microenvironment (TME) consists of a complex network of heterogeneous cell types, including infiltrating immune cells, cancer associated fibroblasts (CAFs), granulocytes, extracellular matrix, endothelial cells, and other stromal components [7]. Among them, CAFs are the main components of stromal cells and can secrete a variety of cytokines to regulate tumor growth and metastasis [8]. Previous study has shown that CAF mediated activation of the Hh-Gli1 pathway is one of the most important molecular mechanisms for promoting the growth, invasion and metastasis of lung cancer [9].

Wenxia Formula (WF) is a TCM formula consisting of four crude herbs: Rheum palmatum L., Aconitum wilsonii Stapf ex Veitch, Panax ginseng C.A. Mey and Angelica sinensis (Oliv.) Diels. The combination of the four herbs utilizes the methods of warming, tonifying, purgation and elimination, and achieves the effect of dispelling cold, restoring yangqi, purgation of stagnation and clearing blood stasis [10]. The results of modern pharmacological studies have shown that the four Chinese herbal medicines have significant antitumor effects [11-14]. Our previous studies have shown that WF can significantly inhibit the proliferation of lung adenocarcinoma cells, induce apoptosis and significantly reduce the expression of PCNA and cyclin D1 [15-18]. However, the specific antitumor components and mechanism of action of WF are not clear.

In order to facilitate the study of the material basis of WF, the WF was extracted with ethanol and oil ether, dichloromethane, ethyl acetate and n-butanol. Four extraction sites were obtained, and the effective antitumor extracts of WF were screened by a cell experiment in vitro. We selected human lung adenocarcinoma cells A549 and H1299, human lung squamous cell carcinoma $\mathrm{H} 520$, and human large cell lung cancer H460 to investigate their antitumor activities in vitro. By calculating the half-inhibitory concentration (IC50), it was found that the ethyl acetate extract from WF (WFEA extract) had the best antitumor activity. In this study, we aimed to illuminate the possible anti-cancer mechanisms of the WFEA extract.

\section{Material and methods}

\section{Animals}

BALB/c Nude mice (6-8 weeks, weighing $20 \pm 2$ g) were purchased from Beijing Vital River Laboratory Animal Technology Co. Ltd (Beijing, China). They were housed in groups of 4-6 per cage in laminar flow hoods in a pathogen-free environment (55 $\pm 10 \%$ humidity, $22 \pm 2^{\circ} \mathrm{C}$ and $12-12 \mathrm{~h} /$ lightdark cycle) with free access to a standard laboratory water and diet. The animals were acclimatized for 1 week before the experiment. The study was approved by the Institutional Animal Care and Use Committee (animal authorization reference number: SCXK2018-0034) at Shandong University of Traditional Chinese Medicine (Shandong, China). Animal welfare and experimental procedures were strictly in accordance with the Guide for the Care and Use of Laboratory Animals (the Ministry of Science and Technology of China, 2006). All efforts were made to minimize the suffering of animals and the number of animals used.

\section{Preparation of WFEA extract}

Rheum palmatum L., Aconitum wilsonii Stapf ex Veitch, Panax ginseng C.A. Mey and Angelica sinensis (Oliv.) Diels were purchased from Shandong Zhonglu hospital. According to the ratio of $9: 12$ : 12 : 6, 9750 g of Panax ginseng C.A. Mey, Rheum palmatum L., Aconitum wilsonii Stapf ex Veitch and Angelica sinensis (Oliv.) Diels dried herbs were weighed. The crushed herbs were extracted ultrasonically twice with $75 \%$ ethanol. After being filtered, the extracts were concentrated to have no alcohol taste, and finally the WFEA extract was obtained. Then, it was extracted three times with petroleum ether, dichloromethane, ethyl acetate and n-butanol, respectively. The extracts were combined and concentrated to obtain petroleum ether partial extract, chloroform partial extract, ethyl acetate partial extract and n-butyl alcohol partial extract, respectively.

\section{Ultra-performance liquid chromatography quadrupole time-of-flight mass spectrometry (UPLC/Q-TOF-MS) analysis}

A XDB-C18 column $(4.6 \times 100 \mathrm{~mm}, 1.8 \mu \mathrm{m})$ was utilized for separation with the column temperature at $35^{\circ} \mathrm{C}$. The standards and samples 
were separated using a gradient mobile phase consisting of (A) acetonitrile and (B) $0.1 \%$ formic acid in water. The flow rate was at $0.5 \mathrm{ml} / \mathrm{min}$. The injection volume of sample was $1 \mu$ l. Mass spectrometry was performed on a Bruker Q-TOF equipped with an electrospray ionization source in a positive ion mode. The temperatures of the electrospray source and desolvation were $200^{\circ} \mathrm{C}$ and $325^{\circ} \mathrm{C}$, respectively. The flow rate of nitrogen was set to $8 \mathrm{l} / \mathrm{min}$. The ESI capillary and cone voltages were set at 3,500 and $30 \mathrm{~V}$, respectively. The MS/MS analysis was performed using a variable collision energy (15-45 eV), which was optimized for each individual constituent.

\section{Cell culture}

The A549 cell line was purchased from Shanghai Institute of Biochemistry and Cell Biology, Chinese Academy of Sciences. Luciferase (LuC) gene-labeled human lung adenocarcinoma A549 cells (A549-Luc) were purchased from Keygen Biotech (Jiangsu China). Human Lung Adenocarcinoma CAFs were purchased from iCell-CRO (Shanghai, China). A549 and A549-Luc were grown in RPMI-1640; the CAFs were cultured in DMEM. Medium was supplemented with $10 \%$ fetal bovine serum (FBS, Gibco, USA), $100 \mathrm{U} / \mathrm{ml}$ penicillin and $100 \mu \mathrm{g} / \mathrm{ml}$ streptomycin in an incubator at $37^{\circ} \mathrm{C}$ with a $5 \% \mathrm{CO}_{2}$ atmosphere.

\section{Xenograft model procedure}

Approximately $4 \times 10^{6}$ cells were suspended in FBS-free medium and mixed with Matrigel $(200 \mu \mathrm{l})$. A549 and A549 plus CAFs (1:1) cells were respectively injected subcutaneously into the mouse right flank. When tumor nodules appeared at the injection site, groups of nude mice injected with A549 plus CAFs cells were divided into 5 groups (WFEA high, WFEA middle, WFEA low, cisplatin (CDDP) and control group). The CDDP group was given an intraperitoneal injection of $5 \mathrm{mg} / \mathrm{kg}$ cisplatin twice a week; the WFEA group was given an intragastric administration of WFEA extract at doses of $400 \mathrm{mg} / \mathrm{kg}, 200 \mathrm{mg} / \mathrm{kg}$ and $100 \mathrm{mg} / \mathrm{kg}$, respectively; the control group and $\mathrm{A} 549$ group were given intragastric administration of equal volume normal saline. Each group was continuously intervened for 5 weeks.

\section{Immunofluorescent staining}

Tissue sections were dewaxed with xylene and dehydrated with graded alcohol. After washing with distilled water, tissue sections were heated at high pressure in ethylene diamine tetraacetic acid (EDTA) antigen repair buffer (PH 8.0) for antigen retrieval. After the slices were cooled at room temperature, sections were incubated with primary antibodies ( $1: 500,4^{\circ} \mathrm{C}$, overnight) and secondary antibodies $\left(37^{\circ} \mathrm{C}, 50 \mathrm{~min}\right)$, respectively. Then paraffin sections were incubated with 4',6-diamidino2-phenylindole (DAPI, Beyotime Biotechnology, China) for $10 \mathrm{~min}$ in the dark. Finally, paraffin sections were sealed in anti-fluorescence. Images were acquired on a laser scanning confocal microscope FV12-IXCOV (OLYMPUS, Japan). Red fluorescence represented the positive expression of genes. The mean optical density was calculated by Image-J to analyze the positive expression.

\section{Immunohistochemistry}

Tissue sections were deparaffinized with $x y$ lene and rehydrated with ethanol, followed by antigen retrieval. After the slices were cooled at room temperature, tissue sections were incubated with primary antibodies $\left(37^{\circ} \mathrm{C}, 2 \mathrm{~h}\right)$ and secondary antibodies $\left(37^{\circ} \mathrm{C}, 30 \mathrm{~min}\right)$, respectively. Following a final washing in PBS, $\alpha$-SMA and FAP signals were visualized with a DAB kit (Sigma, St. Louis, MO, USA), counterstained with hematoxylin and mounted in Kaiser's glycerol gelatine. For semi-quantitative measurements of $\alpha$-SMA and FAP density, the slides were photographed and analyzed using a computer-aided image-analyzing system (Motic Images Advanced 3.2).

\section{Hematoxylin and eosin (HE) staining}

Sections were dewaxed in xylene and rehydrated in a series of alcohol solutions. After washing in distilled water for a short time, the sections were stained in Harris hematoxylin solution for 5 min, washed in tap water and counterstained in eosin-phloxine solution for $2 \mathrm{~min}$.

\section{Quantitative real-time polymerase chain reaction ( $q R T-P C R$ )}

Total RNA was isolated using TRIzol reagent (Invitrogen, USA). The cDNA of Ptch1, Smo and Gli1 was prepared using the RevertAid First Strand CDNA Synthesis Kit. The qRT-PCR analysis was performed using the SYBR Green RT-PCR Kit (Takara Bio, Japan) on an ABI 7500 Fast Real-Time PCR System (Applied Biosystems, USA). $\beta$-Actin was used as the endogenous control to calculate the relative RNA levels. Data were calculated by the

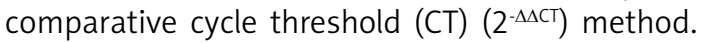
The PCR primer sequences were as follows:

(1) Ptch 1 forward: 5'-GCAACACCTGGACTCAGCACTC-3'; and reverse: 5'-AACCTGTCTCCGTGATAAGTTCC-3';

(2) Smo forward: 5'-GTGCTTATTGTGGGAGGCTACTTC-3'; and reverse: 5'-TTGGCATAGCACATAGTCCCG-3';

(3) Gli1 forward: 5'-GACTTTCTGGTCTGCCCTTTTG-3'; and reverse: 5'-GAGAGAGCCCGCTTCTTTGTTA-3'; 
(4) Shh forward: 5'-AAAGCAGAGAACTCCGTGGC-3'; and reverse: 5'-CACTAACTTGGTGCCACCCT-3';

(5) $\beta$-Actin forward: 5'-GTGACGTTGACATCCGTAAAGA-3'; and reverse: 5'-GTAACAGTCCGCCTAGAAGCAC-3'.

\section{Western blot}

Tumor tissue lysates were prepared using a proper amount of RIPA lysate, and the BCA method was used for protein quantification. Equal amounts of proteins $(20 \mu \mathrm{g})$ of each sample were separated by sodium dodecyl sulfate-polyacrylamide gel electrophoresis and then transferred onto the polyvinylidene fluoride (PVDF) membranes (Millipore, USA). PVDF membrane was blocked in $5 \%$ skim milk at $37^{\circ} \mathrm{C}$ for $1 \mathrm{~h}$, and subsequently incubated at $4^{\circ} \mathrm{C}$ overnight with antibodies against SHH, PTCH1, SMO, GLI1 or ACTIN (all in $1: 1000$ dilutions, Santa Cruz, USA). Next, PVDF membrane was incubated with corresponding horseradish peroxidase-conjugated (HRP)-linked secondary IgG antibodies (anti-rabbit or anti-mouse, 1 : 2000 dilutions, Santa Cruz, USA) for $1 \mathrm{~h}$ at room temperature. The signal was detected with a Super Signal Protein Detection kit (Pierce Biotechnology, USA).

\section{Statistical analysis}

All statistical analyses were performed using SPSS version 22.0 (IBM Corp., IL, USA). Results from each experiment were expressed as the mean \pm standard deviation of three separate experiments. Statistical significance was determined with Student's $t$-test. One-way analysis of variance (ANOVA) was used to perform the be- tween-group comparisons. Statistical significance was set at $p<0.05$.

\section{Results}

\section{Identification of constituents of WFEA extract}

UPLC-Q-TOF/MS was applied to analyze and identify the constituents of WFEA extract. Through the standard comparison, literature review, and disassociation rules, 15 chemical components were identified, and the results are shown in Table and Supplementary Figure S1.

\section{WFEA extract inhibits the tumorigenicity of lung cancer cells}

The antitumor activity in vivo of the WFEA extract was tested in lung cancer xenograft model. The results showed that the weight and volume of xenograft in the control group were greater than those in the A549 group, indicating that CAFs could promote lung cancer cells growth. The weight and volume of xenograft in the WFEA high/ medium/low group were significantly lower than those in the control group, indicating that WFEA extract could inhibit lung cancer cells growth in vivo (Figures $1 \mathrm{~A}-\mathrm{C}$ ). In addition, we found that the body weight of mice in the A549 group, control group and WFEA group showed an increase trend, but there were no significant difference (all $p>$ 0.05). Compared with the control group, the body weight of mice in CDDP group decreased significantly (Figure 1 D).Taken together, these results showed that WFEA extract could inhibit lung cancer cells growth in vivo and was safer than CDDP.

Table I. Identification results of the constituents of WFEA extract

\begin{tabular}{|ccccc|}
\hline No. & Name & $\mathrm{t}_{\mathrm{R}}[\mathrm{min}]$ & {$[\mathrm{M}+\mathrm{H}]^{+}(\mathrm{m} / \mathrm{z})$} & Molecular formula \\
\hline 1 & Catechinic acid & 6.9 & 291.0824 & $\mathrm{C}_{15} \mathrm{H}_{14} \mathrm{O}_{6}$ \\
\hline 2 & Fuziline & 7.7 & 454.2736 & $\mathrm{C}_{24} \mathrm{H}_{39} \mathrm{NO}_{7}$ \\
\hline 3 & Neolin & 8.3 & 438.2787 & $\mathrm{C}_{24} \mathrm{H}_{39} \mathrm{NO}_{6}$ \\
\hline 4 & Chasmanine & 10.9 & 452.2947 & $\mathrm{C}_{25} \mathrm{H}_{41} \mathrm{NO}_{6}$ \\
\hline 5 & $(-)$ epicatechin-3-0-gallate & 11.2 & $\mathrm{C}_{22} \mathrm{H}_{18} \mathrm{O}_{10}$ \\
\hline 6 & Galloyl- $\beta$-D-glucose & 11.8 & $\mathrm{C}_{13} \mathrm{H}_{16} \mathrm{O}_{10}$ \\
\hline 7 & Aconitine & 14.8 & 333.0910 & $\mathrm{C}_{31} \mathrm{H}_{43} \mathrm{NO}_{10}$ \\
\hline 8 & 2,5 -dimethyl-7-hydroxychromone & 15.3 & 590.2874 & $\mathrm{C}_{11} \mathrm{H}_{10} \mathrm{O}_{3}$ \\
\hline 9 & Benzoylaconitine & 16.3 & 191.0680 & $\mathrm{C}_{32} \mathrm{H}_{45} \mathrm{NO}_{10}$ \\
\hline 10 & Benzoylhypacoitine & 17.1 & 604.3028 & $\mathrm{C}_{31} \mathrm{H}_{43} \mathrm{NO}$ \\
\hline 11 & Senkyunolide 1 & 17.5 & 574.2928 & $\mathrm{C}_{12} \mathrm{H}_{16} \mathrm{O}_{4}$ \\
\hline 12 & Chrysophanol & 18.1 & 247.0932 & $\mathrm{C}_{15} \mathrm{H}_{10} \mathrm{O}_{4}$ \\
\hline 13 & Physcion & 20.4 & 255.0618 & $\mathrm{C}_{16} \mathrm{H}_{12} \mathrm{O}_{5}$ \\
\hline 14 & Genistein & 25.5 & 285.0716 & $\mathrm{C}_{15} \mathrm{H}_{10} \mathrm{O}_{5}$ \\
\hline 15 & Ligustilide & 34.1 & 271.0566 & $\mathrm{C}_{12} \mathrm{H}_{14} \mathrm{O}_{2}$ \\
\hline
\end{tabular}


A

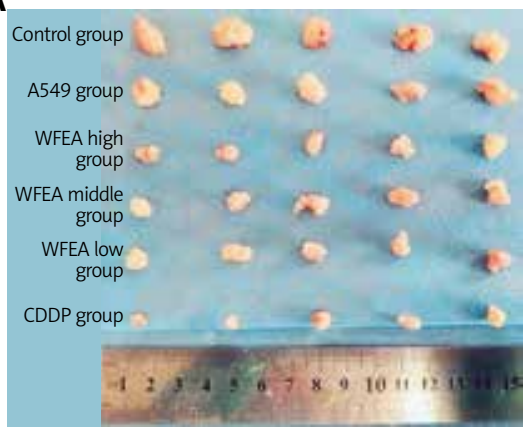

$\mathbf{B}_{0.8}$

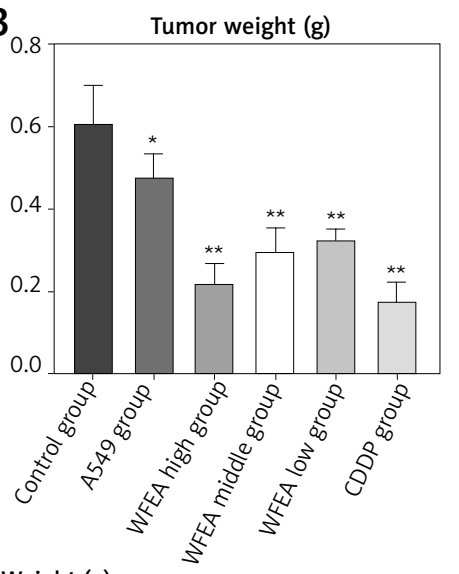

C

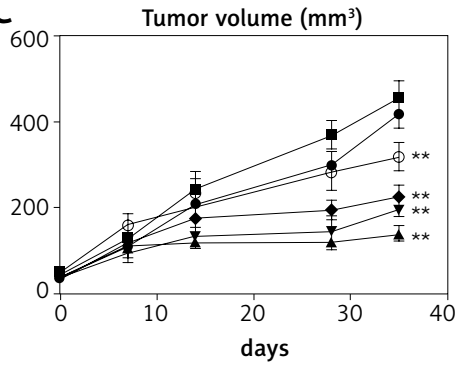

D

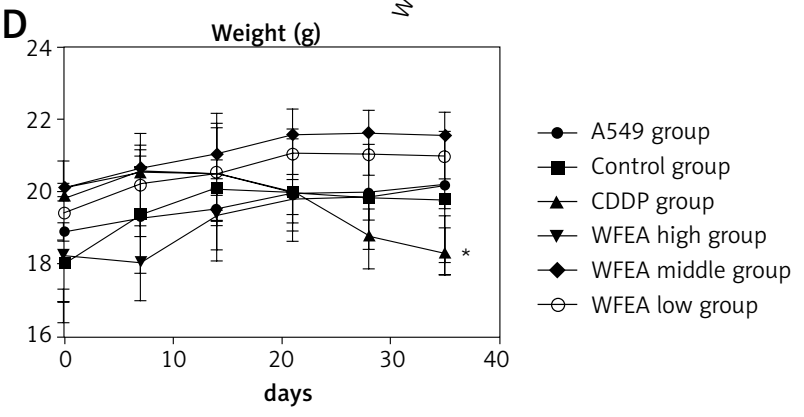

E

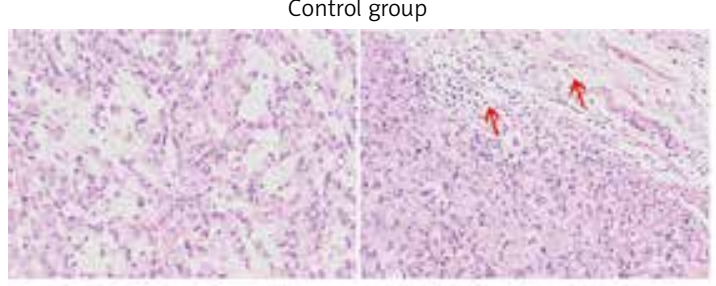

WFEA high group

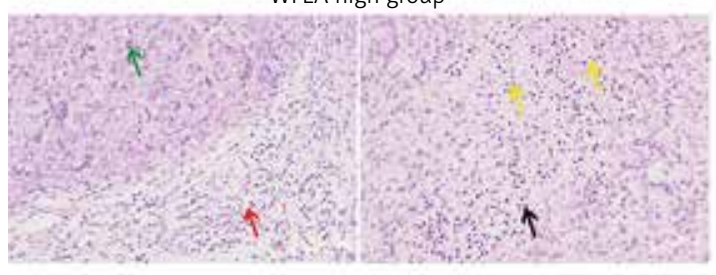

WFEA low group
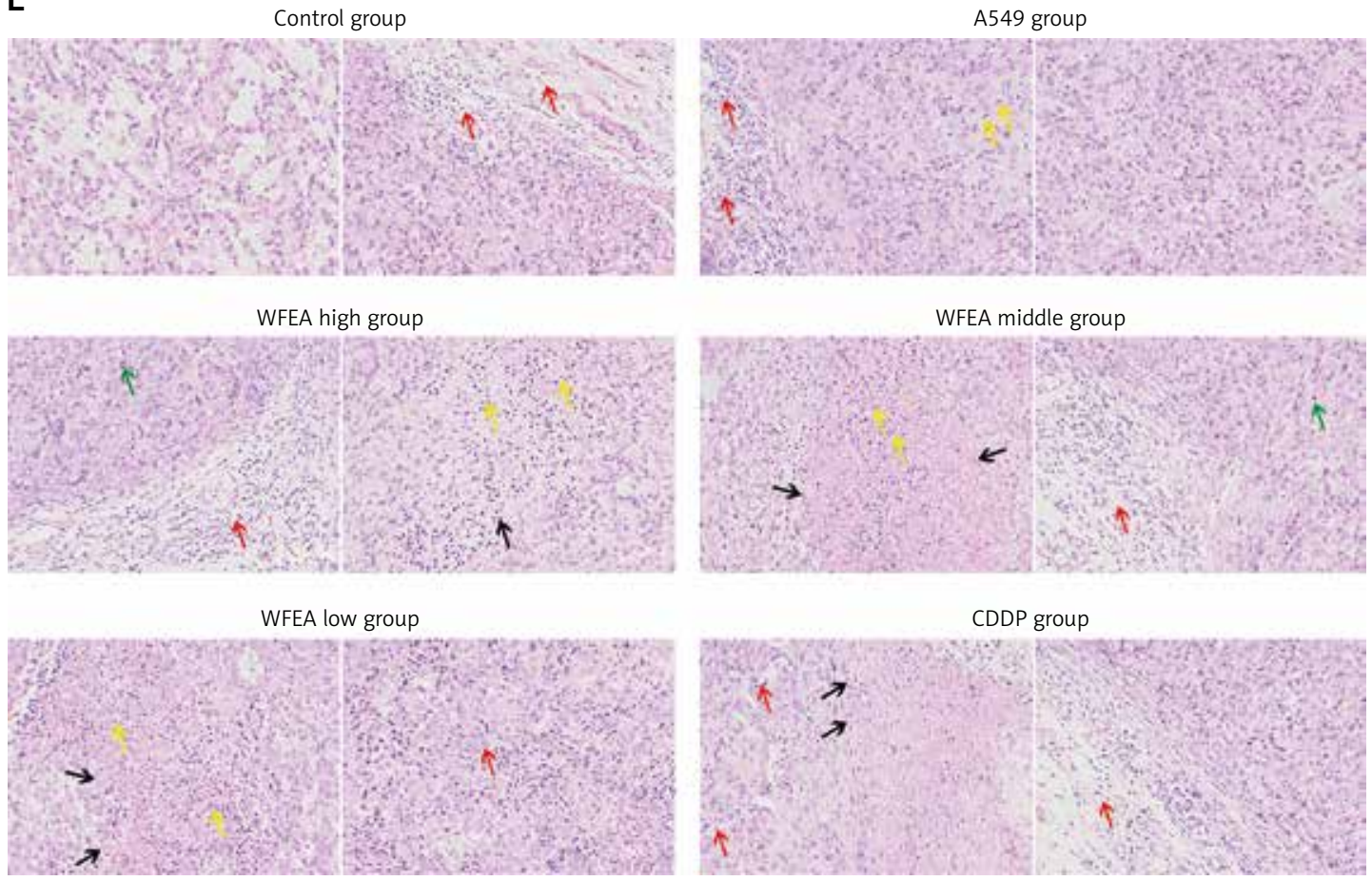

WFEA middle group
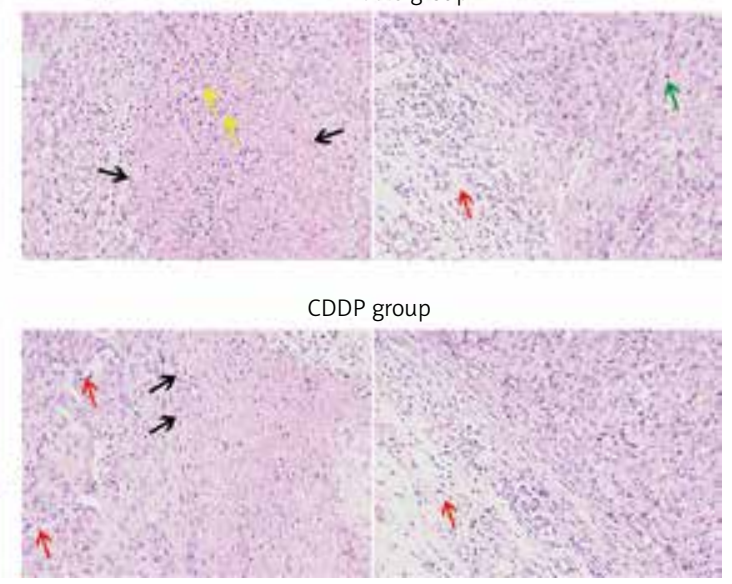

Figure 1. WFEA extract inhibits the tumorigenicity of lung cancer cells. A - optical image showing mouse tumors; $\mathbf{B}$ - weight of tumor in each group; $\mathbf{C}$ - quantification of tumor volume; $\mathbf{D}$ - body weight of mice in each group; E - HE staining $(\times 200)$ of tumors in each group. The black arrow represents nuclear fragmentation or dissolution of necrotic tumors; yellow arrow represents neutrophil infiltration; red arrow represents inflammatory cell infiltration; green arrow represents karyokinesis. ${ }^{\star} p<0.05,{ }^{* *} p<0.01$ (compared with control group); CDDP - cisplatin

\section{WFEA extract decreased expression levels of $\alpha$-SMA and FAP}

The immunohistochemistry results showed that the expression levels of alpha-smooth muscle actin $(\alpha-S M A)$ and fibroblast activation protein
(FAP) in the xenograft of the control group were significantly higher than those in the A549 group $(p<0.05)$, indicating that the activation of CAFs could significantly increase the expression levels of $\alpha$-SMA and FAP (Figure 2). 
A

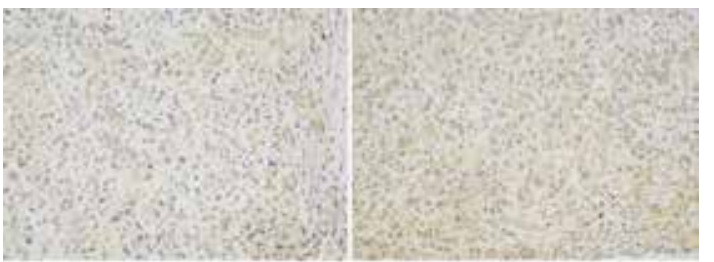

CDDP group

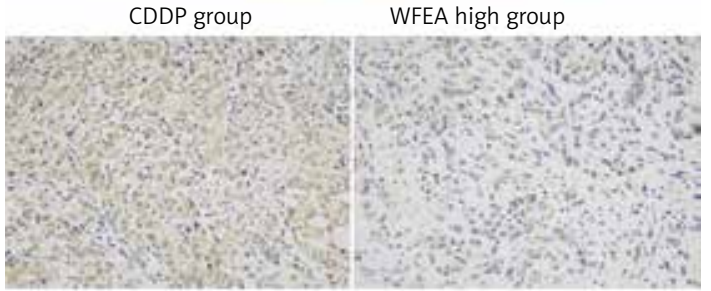

WFEA middle group

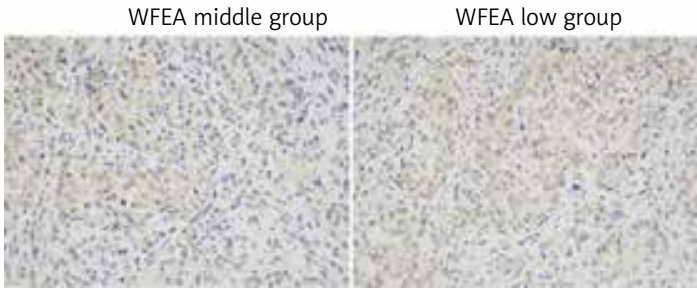

B

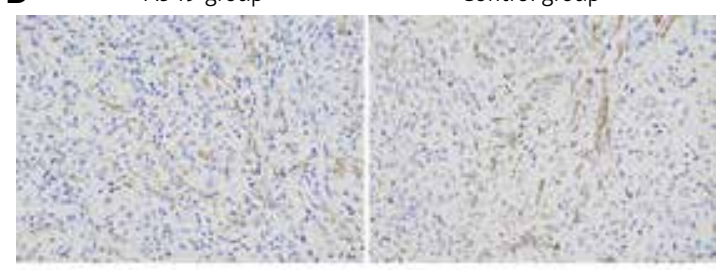

CDDP group

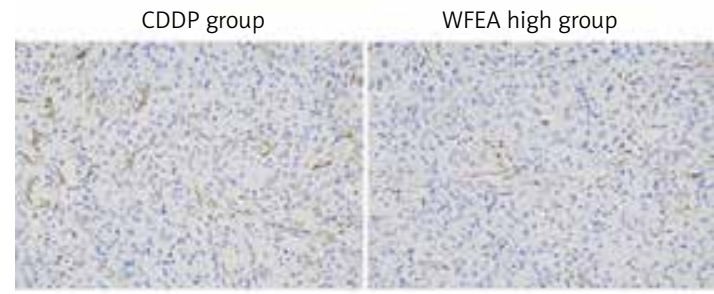

WFEA middle group

WFEA low group

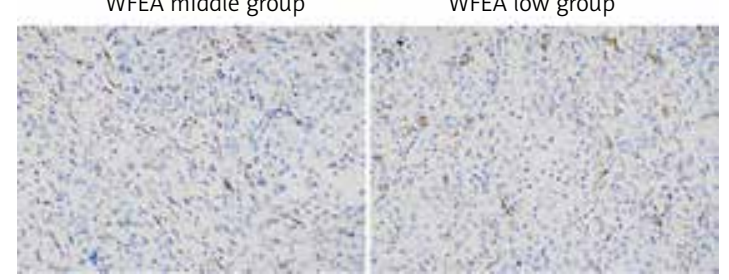

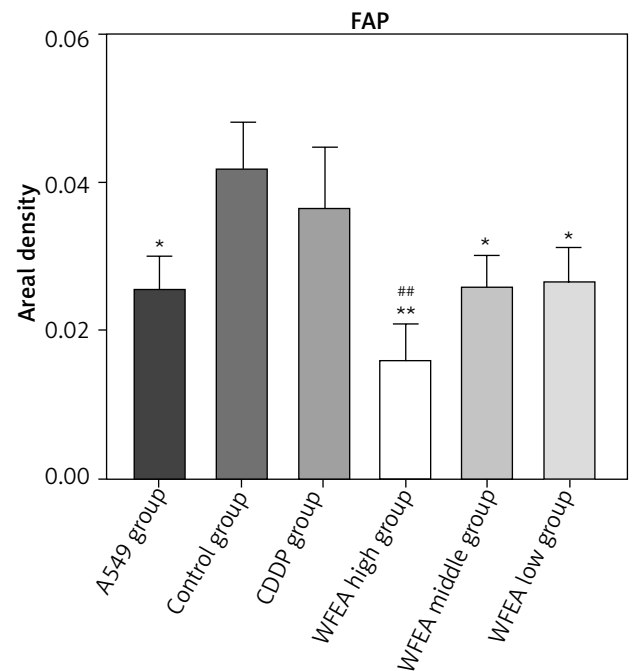

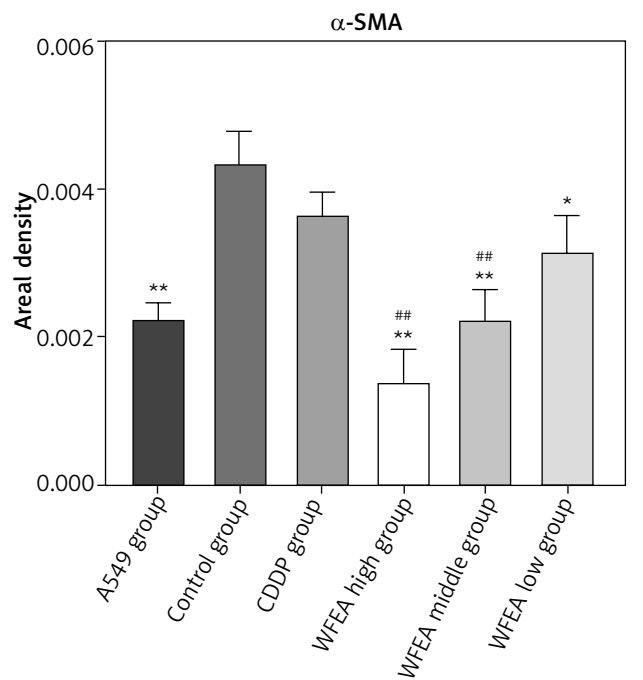

Figure 2. Digital images of xenograft tissue sections after FAP and $\alpha$-SMA immunohistochemical staining. A digital images of xenograft tissue sections after FAP immunohistochemical staining and quantification of the expression of FAP. B - digital images of xenograft tissue sections after $\alpha$-SMA immunohistochemical staining and quantification of the expression of $\alpha$-SMA. ${ }^{*} p<0.05,{ }^{* *} p<0.01$ (compared with control group); ${ }^{*} p<0.05$, $\# p<0.01$ (compared with CDDP group); CDDP - cisplatin

In addition, we found that the expression levels of $\alpha$-SMA and FAP in the WFEA high/medium/ low group were significantly lower than those in the control group, indicating that WFEA extract could significantly decrease the expression levels of $\alpha$-SMA and FAP in the CAF mediated lung cancer xenograft model.

\section{Effect of WFEA extract on E-cadherin and $\mathrm{N}$-cadherin}

In order to illuminate the possible anti-cancer mechanisms of the WFEA extract, we next examined the effects of WFEA extract on marker proteins of epithelial-mesenchymal transition (EMT). The immunofluorescence staining results showed that the ex- 


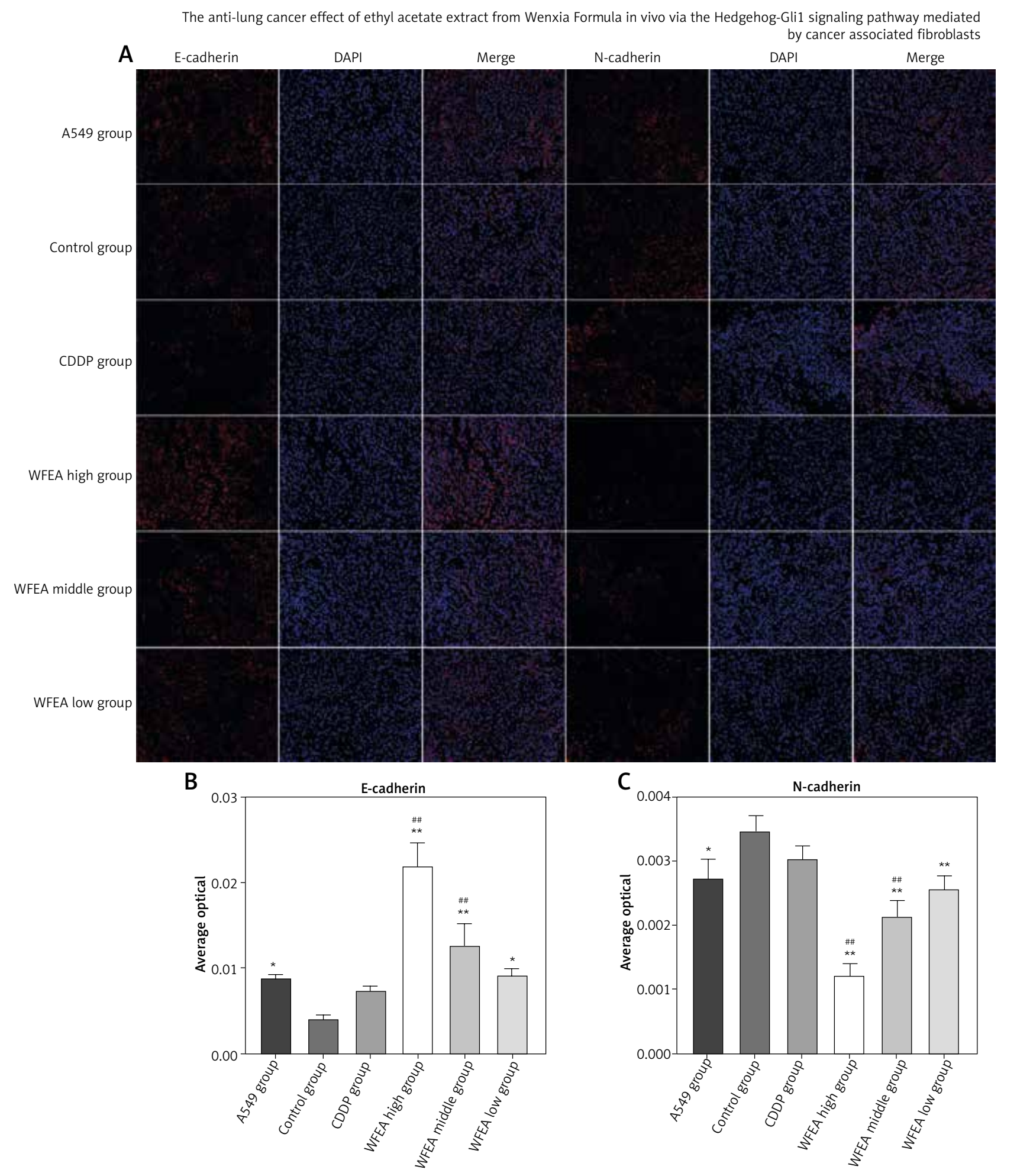

Figure 3. Digital images of xenograft tissue sections after E-cadherin and N-cadherin immunofluorescence staining. A - digital images of xenograft tissue sections after E-cadherin and $\mathrm{N}$-cadherin immunofluorescence staining. Blue fluorescence represents cell nucleus and red fluorescence represents $\mathrm{E}$-cadherin and $\mathrm{N}$-cadherin. $\mathrm{B}-$ quantification of the expression of E-cadherin. $\mathrm{C}$ - quantification of the expression of $\mathrm{N}$-cadherin. ${ }^{*} p<0.05,{ }^{* \star} p<0.01$ (compared with control group); ${ }^{\#} p<0.05,{ }^{\# \#} p<0.01$ (compared with CDDP group); CDDP - cisplatin

pression of E-cadherin in the xenograft of the A549 group and WFEA group was significantly higher than that in the control group (Figure 3).

In addition, we found that the expression of $\mathrm{N}$-cadherin in the xenograft of the A549 group and WFEA group was significantly lower than that in the control group. Taken together, these results showed that WFEA extract could inhibit EMT of lung cancer cells, thereby inhibiting tumor growth and metastasis. 


\section{A}

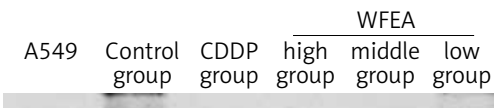

B
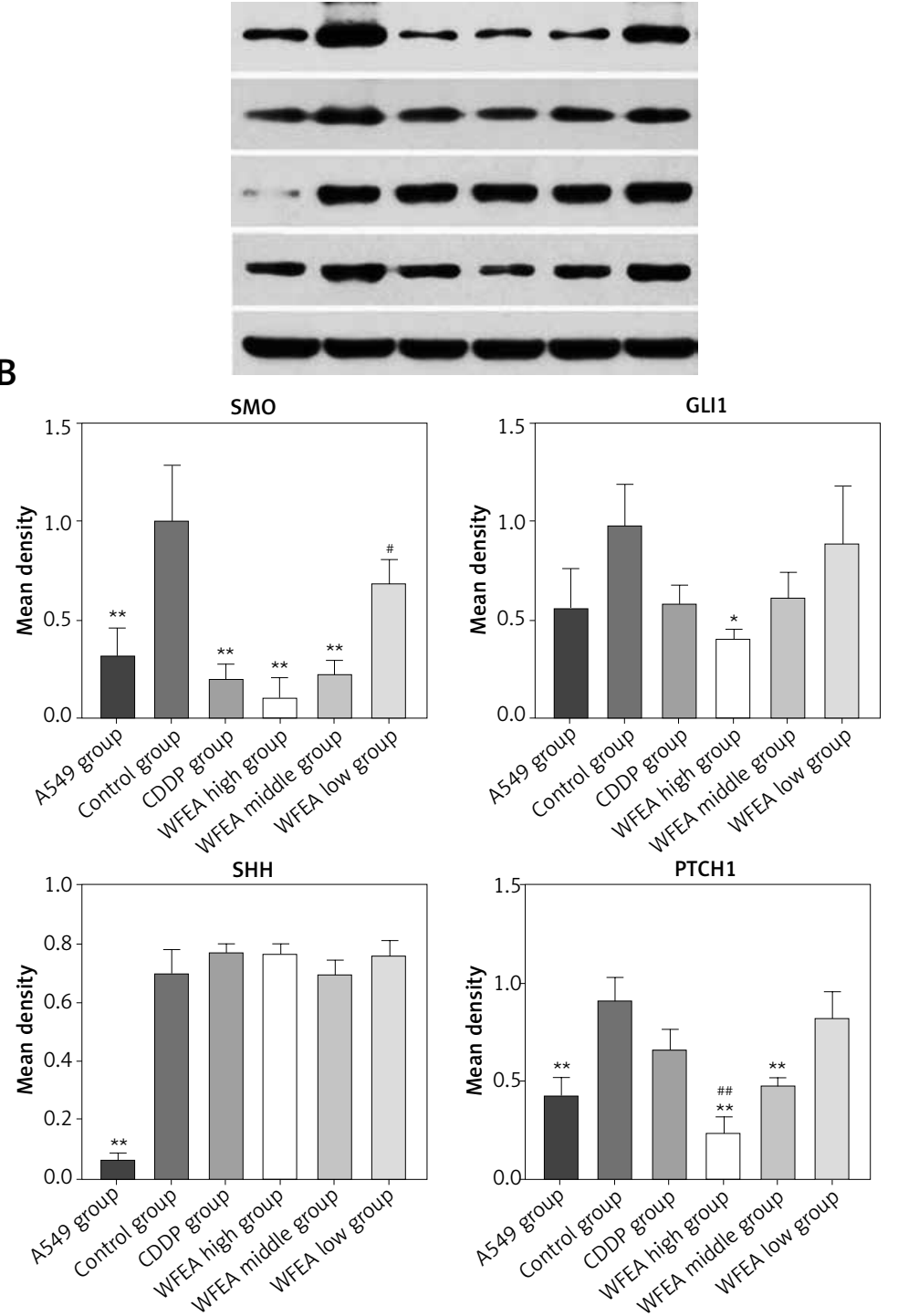

C
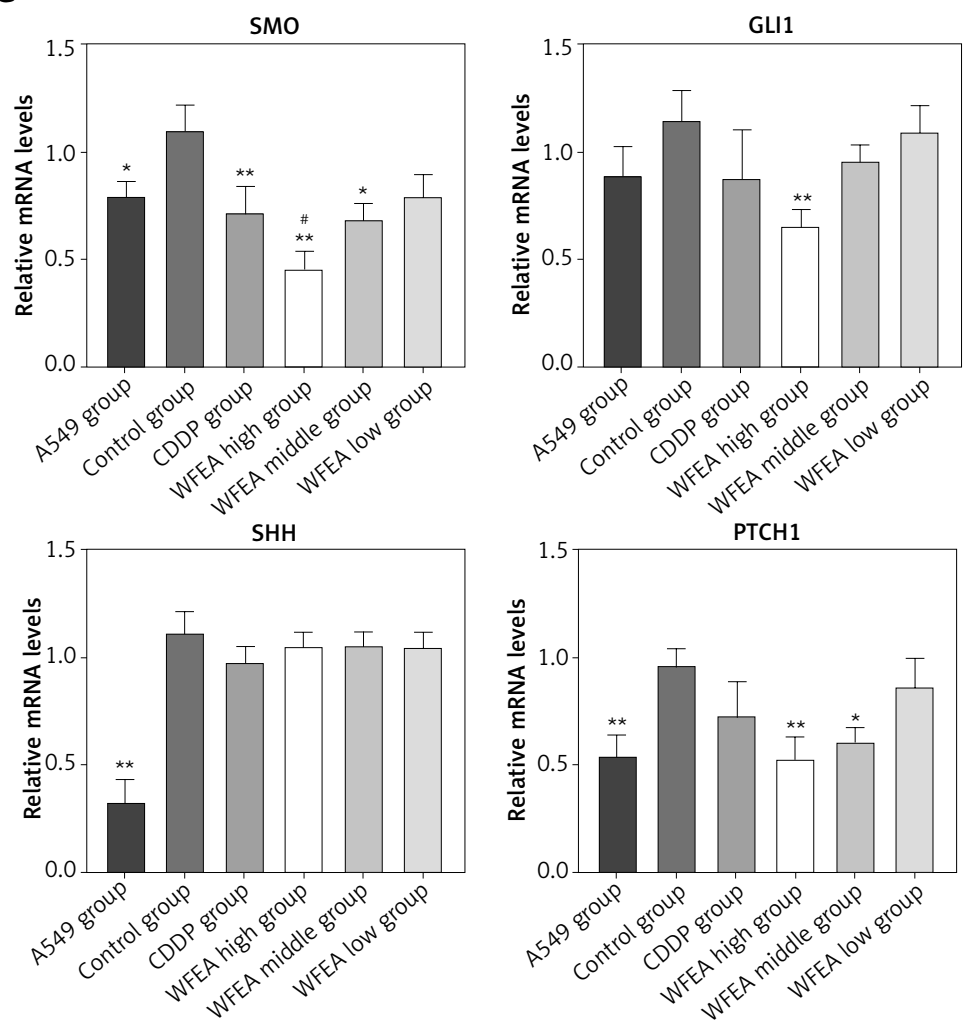

Figure 4. Expression of related proteins and genes (SMO, GLI, SHH and PTCH1) in Hh-Gli1 signaling pathway. A - Western blot analysis of SMO, GLI1, SHH and PTCH1 expression in xenograft. B - quantification of the expression of SMO, GLI1, SHH and PTCH1. C - real-time PCR analysis of Smo, Gli1, Shh and Ptch 1 mRNA in xenograft. * $p<0.05$, ${ }^{* *} p<0.01$ (compared with control group); ${ }^{*} p<0.05,{ }^{\# \#} p<0.01$ (compared with CDDP group); CDDP - cisplatin 


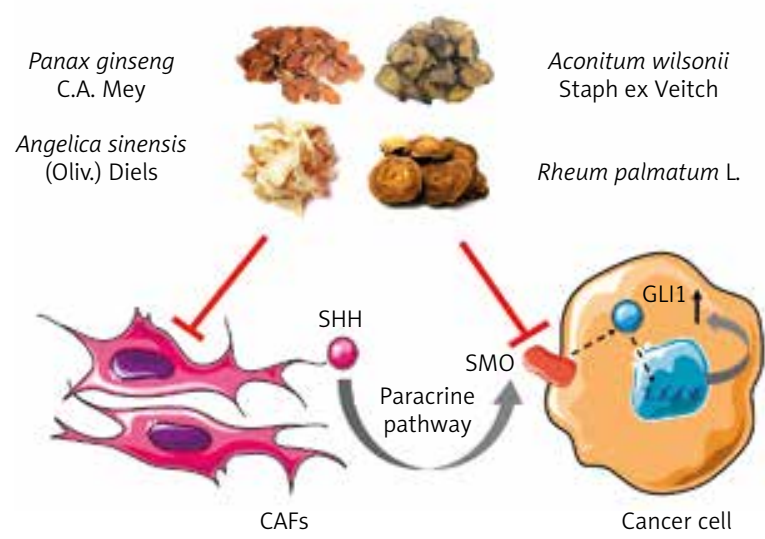

Figure 5. Effect of WFEA extract on Hh-Gli1 signaling pathway mediated by CAF activation. WFEA extract could inhibit the Hh-Gli1 signaling pathway mediated by CAF activation in lung cancer xenograft model

\section{Effect of WFEA extract on Hh-Gli1 signal pathway}

Western blot and qRT-PCR showed that the expression levels of related proteins and genes (SMO, GLI1, SHH and PTCH1) in the Hh-Gli1 signaling pathway of the A549 group and WFEA group were significantly lower than those in the control group (Figure 4). These results indicated that the WFEA extract could inhibit the Hh-Gli1 signaling pathway mediated by CAF activation in the lung cancer xenograft model (Figure 5).

\section{Discussion}

With the development of science and technology, the research on the extraction of effective anti-tumor components from Chinese herbal medicine and the compatibility of Chinese herbal medicine based on the theory of Chinese medicine has attracted increasing attention from the medical community [19]. Some traditional Chinese medicine (e.g. baicalein and Shen-Mai San) has been confirmed to be effective in treating tumors [2022]. WF is a TCM formula consisting of four crude herbs: Rheum palmatum L., Aconitum wilsonii Stapf ex Veitch, Panax ginseng C.A. Mey and Angelica sinensis (Oliv.) Diels. Our previous studies have shown that WF can significantly inhibit the proliferation of lung adenocarcinoma cells, induce apoptosis and significantly reduce the expression of PCNA and cyclin D1 [15-18]. However, the specific antitumor components and mechanism of WF are not clear. In this study, we aimed to illuminate the possible anti-cancer mechanisms of the WFEA extract.

In this study, we found that the weight and volume of the xenograft in the WFEA high/medium/ low group were significantly lower than those in the control group, indicating that the WFEA extract could inhibit lung cancer cell growth in vivo.
These observations were consistent with our previous study [18], which showed that WFEA extract could significantly reduce the tumor volume from the $19^{\text {th }}$ day and alleviate the tumor weight. In addition, we found that WFEA extract had no significant effect on the body weight of mice, while the CDDP group had significant weight loss, indicating that WFEA extract was safer than CDDP.

CAFs are the most important cells in the process of tumorigenesis, which could lead to the initiation and progression of malignant tumors. Among them, $\alpha$-SMA and FAP are the most widely recognized markers of CAFs [23]. In this study, we found that the expression of $\alpha$-SMA and FAP in the tumor tissues of mice co-injected with CAFs and A549 cells (control group) was significantly higher than that of mice injected with A549 alone (A549 group) and the expression levels of $\alpha$-SMA and FAP in the WFEA high/medium/low group were significantly lower than those in the control group. These results indicating that WFEA extract could significantly inhibit the expression of $\alpha$-SMA and FAP and affect the activation of CAFs in tumor tissues.

Epithelial-mesenchymal transition is a key process in tumor growth and metastasis, which includes the decrease of the epithelial cell marker $\mathrm{E}$-cadherin and the up-regulation of $\mathrm{N}$-cadherin and other mesenchymal cell-related markers, so as to reduce the adhesion of cells and increase the invasion and metastasis ability [24]. In this study, we found that the expression of E-cadherin in the xenograft of the A549 group and WFEA group was significantly higher than that in the control group (Figure 4).

In addition, we found that the expression of $\mathrm{N}$-cadherin in the xenograft of the $\mathrm{A} 549$ group and WFEA group was significantly lower than that in the control group. Taken together, these results showed that WFEA extract could inhibit EMT of 
lung cancer cells, thereby inhibiting tumor growth and metastasis.

The Hh-Gli1 signaling pathway is important for inducing embryonic and stem cell development, and the abnormal activation of this pathway is closely related to the development of many malignant tumors [25]. CAF mediated activation of the Hh-Gli1 signaling pathway is one of the most important molecular mechanisms for promoting the growth and metastasis of lung cancer [9]. In this study, we found that the expression levels of related proteins and genes (SMO, GLI1, SHH and PTCH1) in the Hh-Gli1 signaling pathway of the A549 group and WFEA group were significantly lower than those in the control group. These results indicate that WFEA extract could inhibit the Hh-Gli1 signaling pathway mediated by CAF activation.

In conclusion, WFEA extract could inhibit the EMT of lung cancer, and affect the growth and metastasis of tumor tissue. Its mechanism may be related to the CAF mediated Hh-Gli1 signaling pathway. In this study, the antitumor effect and mechanism of WFEA extract were explored in vivo. The next step of our group is to further verify the antitumor effect of WFEA extract and its possible mechanism in vitro.

\section{Acknowledgments}

Meng Wang and Qian-Yu Bi contributed equally to the manuscript and thus share first authorship.

This work was supported by the National Natural Science Foundation of China (number $81573871 \& 81774198$ \& $81703839 \& 81273634)$ and the Open Project of Shandong Co-Innovation Center of Classic TCM Formula (number 2018KFZ03).

\section{Conflict of interest}

The authors declare no conflict of interest.

\section{References}

1. Wong MCS, Lao XQ, Ho KF, Goggins WB, Tse SLA. Incidence and mortality of lung cancer: global trends and association with socioeconomic status. Sci Rep 2017; 7: 14300.

2. Dinglin X, Ding L, Li Q, Liu Y, Zhang J, Yao H. RYBP inhibits progression and metastasis of lung cancer by suppressing EGFR signaling and epithelial-mesenchymal transition. Transl Oncol 2017; 10: 280-7.

3. Ettinger DS, Bepler G, Bueno R, et al. Non-small cell lung cancer clinical practice guidelines in oncology. J Nat Compr Canc Netw 2006; 4: 548-82.

4. Li W-D, Hua B-J. [Preliminary study on the mechanism of Chinese medicine regulating tumor micro-environment "steady state" affecting tumor recurrence]. J Tradit Chin Med 2011; 52: 1891-4.

5. Sun X-g, Lin X-c, Diao J-x, Yu Z-I, Li K. Pi (Spleen)-deficiency syndrome in tumor microenvironment is the piv- otal pathogenesis of colorectal cancer immune escape. Chin J Integr Med 2016; 22: 789-94.

6. Liu Y, Shen T, Hu Y-H, De-Jiao, You F-M. [Thoughts and research methods of Chinese medicine intervention in tumor microenvironment]. Shizhen Guo Yi Guo Yao 2015: 935-6.

7. lyengar NM, Gucalp A, Dannenberg AJ, Hudis CA. Obesity and cancer mechanisms: tumor microenvironment and inflammation. J Clin Oncol 2016; 34: 4270-6.

8. Borriello L, DeClerck YA. [Tumor microenvironment and therapeutic resistance process]. Med Sci 2014; 30: 445-51.

9. Jia Y, Wang Y, Xie J. The Hedgehog pathway: role in cell differentiation, polarity and proliferation. Arch Toxicol 2015; 89: 179-91.

10. Zhang YN, Wu ZC, Yu HY, et al. Chinese herbal medicine Wenxia Changfu Formula reverses cell adhesion-mediated drug resistance via the integrin beta1-PI3K-AKT pathway in lung cancer. J Cancer 2019; 10: 293-304.

11. Luo L-M, Luo Y-N, Jiang Y-N, et al. [Advances in studies on the effective components and mechanisms of ginseng's anti-tumor effects]. Chinese Herbal Medicine 2017; 48: 582-96.

12. Yang N, Xiang L-C, Cao F-J, Li H-L, Wang X-B. [Research progress on the effect and mechanism of emodin on tumor metastasis]. Anti-Tumor Pharmacy 2016; 6: 173-7.

13. Zhang Z-S, Zhang Y-X, Chen S-Y, Li K-X, Zhou J-W. [Effects of the volatile oil of angelica sinensis on the proliferation and cell cycle of glc-82 cells in human lung adenocarcinoma]. Western Chinese Medicine 2018; 31: 14-8.

14. An Z-X, He Y-L, Min W. [Effect of aconite polysaccharide on the expression of MMP-2, MMP-14 in gastric cancer xenografts in nude mice]. Chinese Journal of Experimental Formulations 2019: 11.

15. Ji XM, Ouyang B, Liu H, et al. In vitro and in vivo inhibitory effect of the combination of Wenxia Changfu formula with cisplatin in non-small cell lung cancer. Chin J Integr Med 2011; 17: 908-16.

16. Ji X-M, Ou Y-B, Wang C-L. [Inhibition of proliferation of lung cancer A549 cells and expression of PCNA Rb CyclinDI by Wenxia Formula]. Chinese Archives of Traditional Chinese Medicine 2010; 28: 721-3.

17. Ji X-M, Ou y-B, Li W-H, Wang C-Y. [Effects of Wenxia Formula on protein expression of PCNA, p16 and CyclinD1 in Lewis lung cancer]. Lishizhen Med Mater Med Res 2011: 104-6.

18. Ji XM, Wu ZC, Liu GW, et al. Wenxia Changfu Formula induces apoptosis of lung adenocarcinoma in a transplanted tumor model of drug-resistance nude mice. Chin J Integr Med 2016; 22: 752-8.

19. Tian R, Chen L, Qi F, et al. [Rule analysis and logistic regression model of cancer treatment with traditional Chinese Medicine]. Chinese Medicine Research 2011; 24: 38-41.

20. Mu J, Liu T, Jiang L, et al. The traditional Chinese medicine baicalein potently inhibits gastric cancer cells. J Cancer 2016; 7: 453-61.

21. Wei H, Sun R, Xiao W, et al. Traditional Chinese medicine Astragalus reverses predominance of Th2 cytokines and their up-stream transcript factors in lung cancer patients. Oncol Rep 2003; 10: 1507-12.

22. Lo L-C, Chen C-Y, Chen S-T, Chen H-C, Lee T-C, Chang C-S. Therapeutic efficacy of traditional Chinese medicine, Shen-Mai San, in cancer patients undergoing chemotherapy or radiotherapy: study protocol for a randomized, double-blind, placebo-controlled trial. Trials 2012; 13: 232. 
23. Sugimoto H, Mundel TM, Kieran MW, Kalluri R. Identification of fibroblast heterogeneity in the tumor microenvironment. Cancer Biol Ther 2006; 5: 1640-6.

24. Xue F, Wu X. [Advances in research on the relationship between cancer-associated fibroblasts and EMT]. Journal of Inner Mongolia Medical University 2018: 417-22.

25. Onishi $\mathrm{H}$, Katano $M$. Hedgehog signaling pathway as a new therapeutic target in pancreatic cancer. World J Gastroenterol 2014; 20: 2335-42. 